\title{
Spotlight on the Patient Health Engagement model (PHE model): a psychosocial theory to understand people's meaningful engagement in their own health care
}

This article was published in the following Dove Press journal:

Patient Preference and Adherence

\section{Guendalina Graffigna \\ Serena Barello}

Department of Psychology, EngageMinds HUB Research Center, Università Cattolica del Sacro Cuore, Milano, Italy

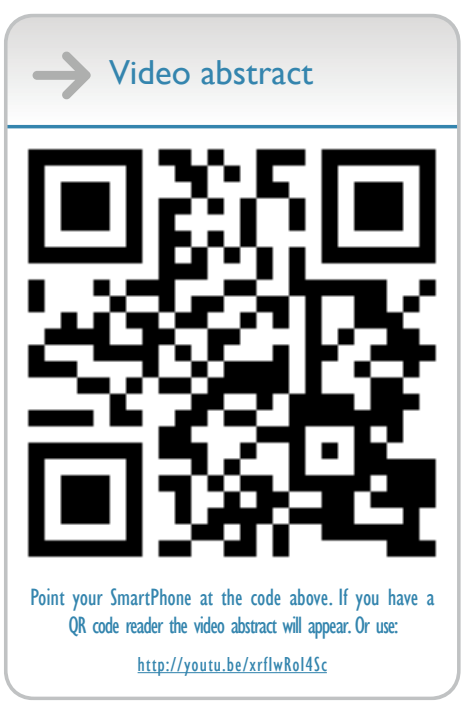

Correspondence: Guendalina Graffigna Department of Psychology, EngageMinds HUB Research Center, L.go Gemelli I, Milano 20123, Italy

Tel +300272346863

Email guendalina.graffigna@unicatt.it

\begin{abstract}
The concept of patient engagement in health care is gaining more and more attention not only in the scientific literature, but also as a requirement in the everyday practices of health care organizations. In general terms, the growing body of literature devoted to patient engagement is mainly inspired by the sociological and public health perspectives, which have generated various theories and models trying to explain how people become active agents in their health and care management. However, theories focusing on the psychosocial dimensions intervening in the patient engagement experience are still limited. This paper proposes a psychosocial perspective on patient engagement and discusses the Patient Health Engagement model, which is an evidence-based psychological theory built on extensive qualitative narrative research and literature analysis aimed at explaining patient engagement and its development in the patients' perspective. The model has been applied to orient patient and professional educational interventions and has contributed to the generation of the first scientific measure of the psychological experience of patients' engagement in their own care (Patient Health Engagement scale). According to this theory, patient engagement is a developmental process that involves the recovered patients' ability to have a life projectuality and goal directedness - even if living with a disease. The paper will also discuss the theoretical origins of this model and will conduct a critical comparison of the theory with the Transtheoretical Model of Change developed by Prochaska and the five-stage grief theory by Kubler-Ross.
\end{abstract}

Keywords: patient engagement, patient empowerment, psychosocial theory, PHE model, emotional factors, psychological dynamics, Transtheoretical Model of Change, five stages of grief theory

\section{Clinical and organizational implications of patient engagement}

Patient engagement in health care is gaining more and more attention not only in the scientific literature but also as a requirement for the everyday practices of health care organizations. ${ }^{1-4}$ In a situation of resource paucity despite the greater demand for health services provision, health care systems are required to "do more with less". ${ }^{5,6}$ In this complex situation, the idea of patient engagement has been advanced as a potential solution to make the health care system more effective and efficient. ${ }^{7}$ First formulated about 10 years ago in the USA, the concept of patient engagement was initially well received by policy makers and health care managers, given the idea that the "individual recipient of care" should be conceived as a crucial actor also in the phases of planning 
and delivery of the health care services themselves. ${ }^{8,9}$ In other words, according to a patient engagement oriented view of health care, patients should be considered one of the crucial human resources in the health care organization and as part of the care team. ${ }^{10}$ Patients not only need to be "put at the center" of the medical action in terms of consideration of their needs and expectations of care, but should also be seen as one of the actors who contribute to the successful accomplishment of the health care organization's mission and actions. ${ }^{11,12}$

The proposal of establishing a true partnership between patients and health care organizations and their professionals in the health care journey is magnified by the concept of patient engagement, which defines the individual not only as a passive recipient of care interventions but also as an actor who should orient such a course of action in a co-creative and participative approach to the management of his/her health and care. ${ }^{13}$ The paradigm of Participatory Medicine is the context in which the concept of engagement grew and assumed concrete meaning. ${ }^{14}$ This approach stresses the importance of sustaining collaborative spaces not only among health care professionals and patients, but also within the overall health care system and the social communities in which patients are embedded: this approach advocates more closely integrated and synergic approaches to patients' care. ${ }^{12}$ This is not to support unregulated self-care without agreement with the responsible health care professional, but - on the contrary - to foster the reciprocal acknowledgment of roles and responsibilities between patients and health care professionals. This mutual awareness and acknowledgment, together with targeted patient education, should be the basis for collaboration and the co-creation of more sustainable and satisfactory health care trajectories.

\section{Factors impacting on patient engagement}

The growing body of literature focused on patients' engagement in their own care is mainly inspired by the sociological and public health perspectives. The most established definitions and models of engagement ${ }^{1,15-18}$ point to the following factors as impacting on the ability of people to be engaged in their health care. Table 1 reports the most established definitions of patient engagement in the current scientific literature.

\section{Patient-related factors}

Some authors ${ }^{17-20}$ have mainly focused on individual-related characteristics of the patient; according to their research, factors such as age, ${ }^{21}$ ethnicity, ${ }^{22}$ level of education, ${ }^{20,23}$ level of income, ${ }^{24,25}$ and also personal dispositions and beliefs about the patient's role in managing health care ${ }^{1,20,26,27}$ may affect individuals' ability to engage with their health care providers along the medical pathway. Moreover, these studies underline the importance of clinical (ie, the gravity of the disease), cultural (ie, patients' cultural-related orientations toward their role in the health care consultation), and social (ie, demographic) characteristics of the patient, along with the characteristics of the prescribed therapeutic regimen (ie, frequency, modes of administering the therapy) as key factors

Table I Main definitions of patient engagement currently available in the scientific literature

\begin{tabular}{|c|c|}
\hline Author(s), year & Definitions of “patient engagement” \\
\hline $\begin{array}{l}\text { Hibbard et al, } \\
2010^{19} \text { (p.1918) }\end{array}$ & Patients' motivation, knowledge, skills, and confidence to make effective decisions to manage their health \\
\hline $\begin{array}{l}\text { Gruman et al, } \\
2010^{17}(p .353)\end{array}$ & $\begin{array}{l}\text { Set of behaviors including two overarching domains: I) "managing health" behaviors, which is both the self-management } \\
\text { of chronic disease and the adoption of healthy behaviors and 2) "managing health care" behaviors, which can be both } \\
\text { patient and "consumeristic" behaviors }\end{array}$ \\
\hline $\begin{array}{l}\text { Carman et al, } \\
2013^{\prime} \text { (p.224) }\end{array}$ & $\begin{array}{l}\text { Set of behaviors by patients, family members, and health professionals and a set of organizational policies and } \\
\text { procedures that foster both the inclusion of patients and family members as active members of the health care team } \\
\text { and collaborative partnerships with providers and provider organizations, so that the desired goals of patient and family } \\
\text { engagement include improving the quality and safety of health care }\end{array}$ \\
\hline $\begin{array}{l}\text { Graffigna et al, } \\
2015^{71} \text { (p.2) }\end{array}$ & $\begin{array}{l}\text { Process-like and multidimensional experience resulting from the conjoint cognitive (think), emotional (feel), and conative } \\
\text { (act) enactment of individuals toward their health management. In this process, patients go through four experiential } \\
\text { positions (disengagement, arousal, adhesion, and eudaimonic project). The unachieved synergy among the different } \\
\text { subjective dimensions (think, feel, act) at each stage of the process may inhibit patients' ability to engage in their care }\end{array}$ \\
\hline Légaré et al, & ["engagement" is] the process of individuals' responsibilization that ensures that clear information leads to the best \\
\hline $2013^{77}$ (p.277) & decision for the person who is seeking the care, thus improving self-management \\
\hline $\begin{array}{l}\text { Mittler et al, } \\
2013^{78}(\text { p.37) }\end{array}$ & $\begin{array}{l}\text { Engaging consumers refers to the performance of specific behaviors ("engaged behaviors") and/or an individual's capacity } \\
\text { and motivation to perform these behaviors ("activation") aimed at gaining health }\end{array}$ \\
\hline $\begin{array}{l}\text { Forbat et al, } \\
2009^{18} \text { (p.84) }\end{array}$ & $\begin{array}{l}\text { A range of ways to conceptualize involvement are used interchangeably in policy and practice without due recognition of } \\
\text { the very different meanings of public consultation, patient/carer involvement in treatment decision making, and patient/ } \\
\text { carer involvement in service design and development }\end{array}$ \\
\hline
\end{tabular}


that could potentially impede patients from gaining the skills necessary to play an active role in the medical journey.

\section{Health professional-related factors}

Other authors ${ }^{12,27-33}$ have highlighted the role of health care professionals and the care team's characteristics in affecting patients' willingness and disposition to become more active in their health care journey. In particular, factors such as the role identity of the health professionals, ${ }^{7,34}$ their set of communicative and relational skills,,$^{20,35,36}$ and their attitude toward the concept of patients' centricity and active engagement in shared decisions about their care process ${ }^{37-41}$ are all indicated as potential factors fostering or impeding the process of patients' engagement in their own care. These authors have indicated the role of professionals' practices and cultures, ${ }^{42-44}$ together with the level of professionals' work motivation, work engagement, and organizational commitment ${ }^{45,46}$ as crucial elements in sustaining their patients' choice to engage actively in their own health management. On the contrary, individual or organizational resistances related to the organizational culture of the health and social services can hinder the process of patients' engagement in their own care. The sensitization and education of health and social practitioners in regard to the concept of engagement is considered a crucial prerequisite for any program promoting the active involvement of patients and their informal/family caregivers.

\section{Organization-related factors}

Other authors have discussed the role of enabling factors connected to the organizational structure, and processes featuring the health care system as an unavoidable requirement for the promotion of patients' engagement in their own care. Specific organizational models, together with the infrastructural features, technological resources available, human resources characteristics, and services' routines and practices are demonstrated as being crucial factors which may sustain or hinder patients' engagement in their own care. Particularly crucial is organizational preparedness (in terms of organizational climate and available resources) and organizational processes: for instance, an institutional vision open to new clinical approaches aimed at fostering the patient's active role and human resources education in this regard. ${ }^{1,12,47-49}$

\section{Lay community-related factors}

Social and community-related factors such as the characteristics of family, informal caregivers, peer networks, and the society as a whole are found to be further facilitators of or obstacles to patient engagement. ${ }^{1,20,50-53}$ The role of communities in sustaining patients' motivation to engage in their own health management is much debated, with particular reference to the role of social media and online communities of peers with the same illness. ${ }^{54-57}$

\section{A health psychology perspective on patient engagement}

In regard to the growing debate on patient engagement in health care, it is interesting that the analyses and evidence related to the psychological dimensions involved in such experience are still limited. ${ }^{16}$

Studies on individual-related factors impacting on patient engagement mainly refer to cultural and sociological determinants, while analysis of the psychological components is mainly focused on cognitive and/or behavioral variables related to such engagement. Based on our research and perspective, only focusing on the behavioral component ${ }^{17,58,59}$ of the patient engagement experience might propose again a passivizing approach to patients' care, as well as it is based on the assumption that a patient has to be "activated" (by someone else) to be effective in self-care. ${ }^{60}$ For instance, Hibbard et al, ${ }^{19}$ in their theory of patient activation, describe engagement as a function of patients' skills, knowledge, and self-efficacy concerning their health and care management. Surprisingly, despite the increasing interest in patient engagement and the growing debate on strategies to foster it, the patients' psychological and emotional experience related to becoming actively engaged in the health care process has been neglected by the scientific community. Scant attention has been paid to the meaning and lived experience of patients when they become engaged in their health care, the extent to which they want (or not) to be engaged, and the drivers of/barriers against its delivery. This lack of an experiencebased modeling of patient engagement may be a missed opportunity to promote the sustainability and effectiveness of services: exploring patients' perspectives on engagement is crucial for supporting care strategies really able to improve engagement in the real world. ${ }^{61,62}$

The discipline of health psychology ${ }^{63,64}$ may support the analysis of engagement in the "patients' eyes". This discipline conducts systematic investigation on how individuals, groups, or organizations enact (or obstruct) health care behaviors and choices. It can offer valuable insights into engagement as a psychosocial and subjective experience, and thus add significantly to the knowledge about engagement and the factors which may sustain or hinder it. 


\section{The Patient Health Engagement model}

The "Patient Health Engagement" (PHE) model was developed by embracing the notions and paradigms of consumer health psychology. It is an experience-based psychological theory based on the systematic and in-depth study of patients' illness experiences by means of narrative qualitative research. ${ }^{16,60}$ According to this theory, patient engagement is a developmental process involving a recovered patient's capacity to plan realistic life projects (ie, meaningful life aims that the patient might generate unless living with the disease condition) and to be goal oriented - even if living with a disease. The process of patient engagement as described by the PHE model involves four developmental phases, namely, blackout, arousal, adhesion, and eudaimonic project. ${ }^{65}$ The model points to the role of the emotional component as the main driver of patients' ability to adjust and adapt to the change of self-role identity involved in the engagement experience. In other words, the concept of engagement implies the patients' personal choice to change their attitude toward the health care system, from passive, to a partner and co-pilot with the health provider of the health care journey. This change in the personal role identity is a function of patients' evolving adjustment and resilience to their health condition.

As said above, the PHE model theorizes a developmental view of patient engagement. It suggests that a fully engaged patient results from a continuous emotional and motivational reframing of his/her health status and his/her role identity. According to the PHE model, becoming engaged in health and care management means being more resilient at the

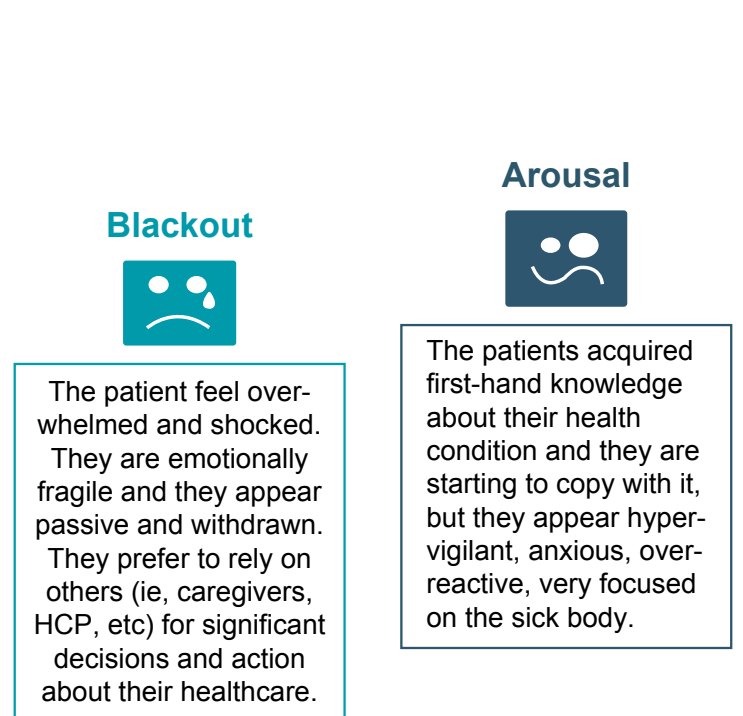

emotional level and effectively adjusting to the health condition and its medical requirements. The final phase of the patient engagement process (eudaimonic project) features an individual with a goal-oriented and more positive approach to health management that is - at this stage - more integrated in the patient's life course. The PHE model theorizes possible trajectories of engagement which are not necessarily linear (a patient may move backward and forward according to critical events in his/her life and disease course). This process also features distinctive ways of interacting and engaging in decisional negotiation between the patient and the health care system that depend closely on the phase of the process through which the patient is passing.

In the following sections, the specific features of each phase of the PHE model will be discussed in depth. These phases are also more deeply explained in some seminal articles by the authors ${ }^{16,34,38,66,67}$ (Figure 1).

\section{Blackout}

This phase of the PHE model mainly occurs when patients experience feelings of psychological vulnerability connected to a critical event, such as a disease diagnosis, a new symptomatology, a disease relapse, and the need to assume new lifestyles in order to manage the health condition. In this phase, patients seem psychologically frozen and feel paralyzed.

In this stage of the PHE model, patients perceive the diagnosis and the requirements for managing the disease as distressing and conflicting with their life habits; for this reason, patients in blackout frequently experience feelings of deep sadness and anger. Consequently, these patients tend

\section{.}

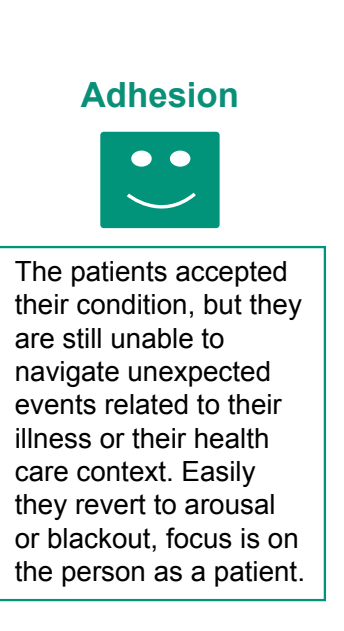

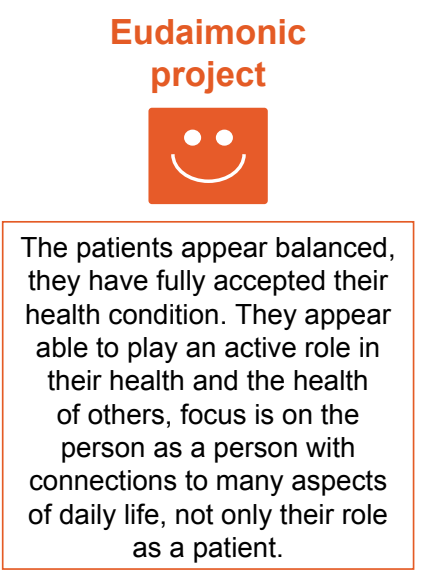

Figure I Description of the phases featuring the PHE model. Abbreviation: PHE, Patient Health Engagement. 
to be passive toward their health care providers. To overcome the blackout phase, patients primarily need emotional and psychological support in order to adjust to their new health condition and develop a new sense of agency and control and sense of effectiveness concerning their health management.

\section{Arousal}

Patients in arousal have acquired an initial awareness of their health condition, but still have superficial knowledge about how to manage it in an effective way. They are not able to adapt to it and to consider their new health status as part of their daily lives. These patients often report that they are hypervigilant over their body and body signals. Every unexpected change in their body status causes emotional alarm and overwhelming emotional responses. In this position, health care providers are crucial points of reference for patients. They must also support them in managing their illnesses and coping with illness, thus preventing care dropouts (that are particularly frequent in this phase).

\section{Adhesion}

When patients succeed in the process of emotional regulation and coping with the illness condition, they experience the third phase of the PHE model: adhesion. In this phase, patients have developed a good acceptance of their disease and have overcome the major psychological stress connected to the disease onset. Moreover, they report being more and more aware of their health status and of its impact on their lives and life habits. In this phase, moreover, patients are increasingly knowledgeable about how to effectively manage their disease and treatment.

However, patients undergoing the adhesion phase are not fully autonomous in their disease management in terms of medical prescription (both life habits and therapeutic regimens), and they frequently experience trouble when something in their life context changes (ie, going on holiday, working life changes, and so on). This happens because patients in this phase are not totally aware of the reasons behind the medical prescriptions; they appear to be formally compliant with their health providers' requirements, but risk failing when some variables in their life change. As a consequence, patients in this phase need to be assisted by an expert figure who can help them maintain correct health behavior even in stressful or atypical situations. Although patients in this phase still tend to rely frequently on their health care professionals' advice and support, they also start to perceive the importance of playing an active part in their health care journey. This initial awareness about their role identity, not only as patients but also as persons who are active partners in the medical course, is a crucial antecedent of the last phase of the PHE model: the eudaimonic project phase.

\section{Eudaimonic project}

When patients become totally aware of their disease and its implications in terms of changed life habits and therapeutic requirements, they are able to become active agents in reaching a positive and satisfying quality of life, unless living with a disease condition. Patients experiencing the eudaimonic project phase - the last one described by the PHE Model have elaborated and accepted "patient identity" as one of the many features in a person's life. In the "eudaimonic project" phase, patients are able to integrate - in a more synergic manner - their health and disease management into their life goals. This reframed perspective of patients toward their health condition makes them able to embrace a more positive and satisfactory approach to their life.

Patients experiencing this phase of the PHE model are also more effective in health information-seeking processes and understanding the rationale behind the medical prescription (differently from the previous phases). Finally, in this phase, patients are able to "activate" the health care professionals when needed, in order fully to respond to their needs and well-being expectations. Health care practitioners are thus considered as reliable allies, and patients see themselves as active members of the care team. Finally, patients in the eudaimonic project phase are also able to become advocates of patients' rights and to play the role of peer experts in patient associations and advocacy groups, or in co-producing services at a policy level.

\section{The PHE model and its links with other health psychology models}

The PHE model discussed in this article has its theoretical roots in both behavioral change and adaptation to change theories and their related constructs in the discipline of health psychology. Inspired by these epistemological roots, the PHE model seeks to furnish a psychosocial processual theory aimed at explaining how people adjust emotionally to their health status and reframe their self-conception, shifting from playing a passive role (asking for a paternalistic approach to care) to considering themselves as authors and owners of their own health and care, ready to act as partners in the health care system in terms of playing an active role in managing their own health. Thus, the PHE model emphasizes the role of the emotional and dynamic components of the engagement 
process in affecting patients' cognitions and behaviors related to their health management. Changes imposed by the new health condition on patients' daily lives may cause emotional upheavals, which often determine the loss of meaning in life, self-confidence, and motivation to go on and adapt to the change. Thus, the dynamic process of psychological adaptation to such a crucial event requires not only a new cognitive mindset and new behavioral skills, but also a complex process of emotional adjustment and self-identity elaboration able to sustain the patient in the reconfiguration of a self-image that does not overlap with the diagnosis but includes it. These emotional and psychodynamic components of the PHE model are - from our perspective - its distinctive features which differentiate and make this psychological approach complementary to other theoretical frameworks of patient engagement rooted in other disciplinary traditions (ie, sociology, public health, health policy) which mainly focus on patients' behavioral activation ${ }^{1,17,19,59}$ or cognitive or emotional reframing processes. ${ }^{41,68}$ The dynamic nature of the emotional adjustment to the disease onset has been well described by the adaptation models developed in the field of health psychology. The term "adaptation theories" is used to denote a group of theories and models that try to explain the psychological process of adjustment that takes place whenever people are faced with situations that require them to undertake a major revision of their assumptions about the world and adapt to those situations (eg, the onset of a disease). One of the most established among these models is the one developed by Kubler-Ross, ${ }^{69}$ who propounded a five-stage theory describing the psychological reactions of terminally ill individuals to their disease: that is, denial-dissociationisolation, anger, bargaining, depression, and acceptance. The stage theory of grief has been generalized to a wide variety of adaptation processes, including patient reactions to chronic diseases. The scientific definition of recurrent patterns of individuals' adaptation to change is undoubtedly of great applied importance because it augments the comprehension of how individuals psychologically react to the life changes with which they are coping. The above-outlined five stages of emotional change theory feature a process that ranges from the astonishment and emotional chaos caused by the critical event to a phase of emotional integration which allows the patient to adapt to the situation and the required changes. The processual model proposed by Kubler-Ross well describes the emotional elaboration and meaning-making process that patients experience when coping with disease. The Kubler-Ross model was a fundamental root for the PHE model. Particularly, some connections can be found with the emotional reactions described in the different phases of PHE model (ie, denial, alert, acceptance, elaboration). However, the PHE model does not only describe the emotional adjustment to the disease and the diagnosis, but also tries to describe how the self-concept reconfiguration (ie, from a healthy individual to an ill patient, to an engaged individual throughout the health care course) happens, so that the patient can embrace a more proactive role in his/her health care journey. However, this implies that the patient autonomously takes the decision to change his/her attitude toward the health care journey by opting for a more proactive role in not only the determination of his/her health outcomes, but also the definition and planning of health care services and pathways. Thus, besides an emotional process of adjustment to the health condition, the PHE model also implies patients' motivation to change attitudes and behaviors related to health management.

In the domain of health psychology, social cognition models have been widely applied to explain patients' behavioral change. The Transtheoretical Model of change is certainly the one that has best theorized the evolution of individuals' motivation and decision making in regard to health behaviors, and it is thus, to some extent, aligned with the PHE model, at least in its theoretical goals. The Transtheoretical Model - by embracing theoretical contributions from various disciplinary domains of psychology - was the first to stress the complexity and the stage process of change in health behavior. In particular, the Transtheoretical Model maintains that individuals change their health behaviors in different stages. They pass from not being concerned to change their behaviors (pre-contemplation stage) to starting to think about them (contemplation) to finally enacting them (action stage) and fully engaging in those behaviors in the long run (maintenance). ${ }^{70}$ The model furnishes strategies to guide the individual through five stages to long-term maintenance, and it includes self-efficacy as a key construct. This model unveiled the complexity of the patients' decisional dynamics when changing their health-related behaviors and described 10 cognitive processes which play a role in sustaining patients' behavioral change toward a more active and aware role in managing health. However, the main focus on self-confidence, on the locus of control, and the decisional balance to nurture the decision for a behavioral change leave less prominence to the emotional and motivational components of that decision. Although Prochaska's model mentions the role of patients' (cognitive) awareness about the need to change behaviors, it does not refer to the role of illness emotional elaboration and psychological adaptation to the change (aspects widely addressed, as said above, by the 
PHE model). Furthermore, this model is not directly linked to the concept of patients' engagement and participation in health care, but rather on the decision making for health prevention and self-management.

\section{The PHE application to clinical practice \\ The PHE scale (PHE-s)}

There is increasing debate on the importance of giving voice to patients regarding their health and care experience and the importance of collecting patient-reported experience measures. ${ }^{62}$ Among the patients' experiences to be recounted and reported, the level of patient engagement is regarded as a key indicator of the quality of patients' experience, and in particular, as a predictor of how patients will be able to maximize their care pathway. Adopting a scientific measurement of engagement, thus being sensitive to this experience variation among individuals and clinical situations, is key for improving clinical practice. The PHE-s ${ }^{71}$ has been developed to advance theory in this field (Figure 2). The scale, developed according to the PHE model theoretical framework, is the first international tool devoted to assess the psychological experience of engagement, and it has robust psychometric proprieties. This quantitative scale only comprises five items and has the peculiarity (in che senso? [in English "peculiar" = "unusual/strange/eccentric"]) of adopting an ordinal structure, so that the patient can describe his/her experience along a continuum of engagement featuring four main positions (see the PHE model). The items were developed based on both systematic analysis of the literature and wide-ranging qualitative studies on chronic patients' engagement in their health care management. ${ }^{33,38,65}$ The phrasing of each item was formulated according to the language and terms used by patients in the qualitative phase that oriented the scale development and was then cognitively tested in the pilot phase of the scale generation. This ensured that the items featuring the PHE-s were well understood by the general population. The scale was designed to be self-administered by the patient,

\begin{tabular}{|c|c|c|c|c|c|c|c|}
\hline Think & about my health st & tatus & & & & & \\
\hline \multirow[t]{2}{*}{1} & $\begin{array}{c}\text { I feel } \\
\text { pyschologically } \\
\text { frozen and } \\
\text { blocked }\end{array}$ & & I feel alert & & I am aware & & I feel positive \\
\hline & $\mathrm{O}$ & $\mathrm{O}$ & $\mathrm{O}$ & $\mathrm{O}$ & $\mathrm{O}$ & $\mathrm{O}$ & $\mathrm{O}$ \\
\hline \multirow[t]{2}{*}{2} & I feel dazed & & I feel worried & & I am conscious & & I feel peaceful \\
\hline & $\mathrm{O}$ & $\mathrm{O}$ & 0 & $\mathrm{O}$ & $\mathrm{O}$ & $\mathrm{O}$ & $\mathrm{O}$ \\
\hline \multirow[t]{2}{*}{3} & $\begin{array}{l}\text { When I think } \\
\text { about my illness, } \\
\text { I feel } \\
\text { overwhelmed by } \\
\text { emotions }\end{array}$ & & $\begin{array}{c}\text { I feel anxious every time a } \\
\text { new symptom arises }\end{array}$ & & $\begin{array}{l}\text { I am used to my } \\
\text { illness }\end{array}$ & & $\begin{array}{l}\text { I find my life meaningful } \\
\text { despite my illness }\end{array}$ \\
\hline & $\mathrm{O}$ & $\mathrm{O}$ & $\mathrm{O}$ & $\mathrm{O}$ & $\mathrm{O}$ & $\mathrm{O}$ & $\mathrm{O}$ \\
\hline \multirow[t]{2}{*}{4} & $\begin{array}{l}\text { I feel very } \\
\text { discouraged due } \\
\text { to my illness }\end{array}$ & & $\begin{array}{l}\text { I feel anxious when I try to } \\
\text { manage my illness }\end{array}$ & & $\begin{array}{l}\text { I have adjusted to my } \\
\text { illness }\end{array}$ & & $\begin{array}{l}\text { I feel optimistic about my } \\
\text { future }\end{array}$ \\
\hline & $\mathrm{O}$ & $\mathrm{O}$ & $\mathrm{O}$ & $\mathrm{O}$ & $\mathrm{O}$ & $\mathrm{O}$ & $\mathrm{O}$ \\
\hline \multirow[t]{2}{*}{5} & $\begin{array}{l}\text { I feel totally } \\
\text { oppressed by my } \\
\text { illness }\end{array}$ & & $\begin{array}{l}\text { I feel upset when a new } \\
\text { symptom arises }\end{array}$ & & $\begin{array}{l}\text { I have accepted my } \\
\text { illness }\end{array}$ & & $\begin{array}{l}\text { I have a sense of purpose } \\
\text { despite my illness }\end{array}$ \\
\hline & $\mathrm{O}$ & $\mathrm{O}$ & $\mathrm{O}$ & $\mathrm{O}$ & $\mathrm{O}$ & $\mathrm{O}$ & $\mathrm{O}$ \\
\hline
\end{tabular}

Figure 2 Description of the PHE-s: introduction and items. Abbreviation: PHE-s, Patient Health Engagement scale. 
who was required to select one response option for each item (line). For more details on administration and scoring, see the previous papers by the authors of this study. ${ }^{71,72}$

Although the PHE model described four engagement positions, the PHE-s features a seven-point scale to facilitate the patients' responses and to avoid social desirability biases. To make a patient free to rate himself, positioning in an intermediate position between two stages of engagement might facilitate a more accurate patient response. According to the ordinal nature of the PHE-s, the median score is considered the more reliable index to calculate the final patients' scoring. ${ }^{72}$ To obtain the final PHE-s position, the median of the row PHE-s scores should be calculated. According to the score obtained, each respondent can be allocated to one of the four engagement positions as described in the PHE model. The scale is based on the assumption that the score obtained by the patients should reflect the actual patient engagement level.

With its brevity and simplicity, the scale was developed to be easily used in "real-world" clinical contexts in order to give voice to patients about their engagement experience and to offer a structured and reliable measurement for clinicians to tailor their communication strategies with the characteristic of the single patient. ${ }^{72}$ Figure 2 shows the PHE-s items. PHE-s is at present validated in various languages and cultural contexts. Validations in Italian, ${ }^{71}$ Chinese, ${ }^{73}$ and Spanish $^{74}$ have already been published.

\section{The PHE model in action}

As mentioned earlier, the PHE model may be used as a psychological framework not only to assess the engagement of patients, but also to detect their needs and expectations to improve along the engagement journey. In particular, corresponding to each engagement position featured by the PHE model are specific needs of support and psychological counseling. The PHEinACTION intervention protocol has been developed specifically to support patients in their psychological journey of engagement. ${ }^{75}$ The PHEinACTION intervention protocol is an individual coaching intervention. It consists of two face-to-face consultations with the clinician (eg, nurse, physician, general practitioner, psychologist) sustained by a set of home-based assignments and exercises personalized according to the fours phases of engagement.

The key features of the PHEinACTION intervention are the following:

1. It is a personalized engagement counseling based on assessment of the level of engagement performed with the PHE-s. The assessment of the level of engagement is considered crucial not only because it personalizes the counseling, but also because it is a first occasion of selfawareness where the patient comes to terms with his/her engagement experience and also an occasion of goal setting in terms of the level of engagement to be achieved.

2. The home-based assignments and exercises proposed are personalized according to the level of patient engagement. Moreover, they are multi-componential, targeting not only the cognitive (health literacy) and behavioral (self-management), but also the emotional resilience of patients.

3. The protocol is quite simple and structured, and it may be adopted in autonomy by the patient, albeit with the support and supervision of a dedicated health care professional.

For further details, the authors of this study refer to previously published papers. ${ }^{76}$

\section{Conclusion}

To sum up, the psychological adaptation and elaboration have an essential role in how patients can (or cannot) cope with their illness, thus influencing their possibility and choice to become effectively engaged in their health care. The transition from a passive approach to active behaviors in managing health and care is based on a complex process of meaning-making and self-identity elaboration, which is primarily emotional and psychodynamic. The subjective, and sometimes irrational, perceptions that individuals have about themselves and the subjective viewpoint through which individuals define the criteria to evaluate their quality of life are - in our opinion - fundamental for enabling the process of engagement and should be included in real-life health engagement models. The value of a psychosocial theory - like the one proposed by the PHE model - is judged not only by its explanatory and predictive power, but also by its applied potential to guide psychosocial changes. In this regard, the PHE model has demonstrated quite an interesting predictive power on the level of patients' adherence to medical prescriptions ${ }^{16}$ and on the level of patients' empowerment in self-management. ${ }^{66}$ These features of the PHE model make this theory particularly interesting also for both service delivery and policy making, because it may function as a "compass" to orient and personalize clinical actions and policy making initiatives based on the patient or population level of patient engagement (and, on the contrary, risks of disengagement). The PHE model has the strength of offering a processual explanation of the psychosocial dynamics occurring when a patient becomes engaged in his/her own 
health care and recognizes that being fully engaged strongly depends on individuals' choice and disposition to play an active role in the care team. For these reasons, the model according to the current literature - does not maintain that more engagement is always the best. On the contrary, effective patient engagement occurs when the health care system recognizes and addresses the patient's unique needs specific of each patient engagement phase. However, the shortcoming of the model is that it does not measure actual patient engagement behaviors. Future research should, therefore, illuminate the heuristic and applicative power of the PHE model, above all, in predicting patient engagement behaviors. Nevertheless, adoption of a psychosocial approach for defining and measuring patient engagement promises to greatly enhance our understanding of how people can decide to change their role in the health care journey in favor of engagement.

\section{Disclosure}

The authors report no conflicts of interest in this work.

\section{References}

1. Carman KL, Dardess P, Maurer M, et al. Patient and family engagement: a framework for understanding the elements and developing interventions and policies. Health Aff (Millwood). 2013;32:223-231.

2. Dentzer S. Rx for the "Blockbuster Drug" of patient engagement. Health Aff. 2013;32(2):202.

3. Blanton K. The patient engagement prescription. N C Med J. 2015; 76(3):157-160.

4. Pelletier LR, Stichler JF. Action brief: patient engagement and activation: a health reform imperative and improvement opportunity for nursing. Nurs Outlook. 2013;61(1):51-54.

5. Suhrcke M, Nugent RA, Stuckler D, Rocco L. Chronic Disease: An Economic Perspective. London: Oxford Health Alliance; 2006.

6. Gordon JE, Leiman JM, Deland EL, Pardes H. Delivering value: provider efforts to improve the quality and reduce the cost of health care. Annu Rev Med. 2014;65:447-458.

7. Coulter A. Leadership for patient engagement. King's Fund. 2012:20.

8. Barello S, Graffigna G, Savarese M, Bosio AC. Engaging patients in health management: towards a preliminary theoretical conceptualization. Psicologia della Salute. 2014;3(3):11-33.

9. Graffigna G, Barello S, Triberti S. Patient engagement: A consumercentered model to innovate healthcare. Berlin: Walter de Gruyter $\mathrm{GmbH}$ \& Co KG; 2016

10. Vahdat S, Hamzehgardeshi L, Hessam S, Hamzehgardeshi Z. Patient involvement in health care decision making: a review. Iran Red Crescent Med J. 2014;16(1):e12454.

11. Graffigna G, Barello S, Riva G, et al. Promozione del patient engagement in ambito clinico-assistenziale per le malattie croniche: raccomandazioni dalla prima conferenza di consenso italiana [Promotion of patient engagement in the clinical-care setting for chronic diseases: recommendations from the first Italian consensus conference]. Recenti Prog Med. 2017;108:455-475. Italian.

12. Graffigna G, Barello S, Riva G, et al. Fertilizing a patient engagement ecosystem to innovate healthcare: toward the first Italian Consensus conference on patient engagement. Front Psychol. 2017;8:1-6.

13. Hardyman W, Daunt KL, Kitchener M. Value co-creation through patient engagement in health care: a micro-level approach and research agenda. Public Manag Rev. 2015;17(1):90-107.
14. Dyson E. Why Participatory medicine? J Particip Med. 2009;1:1-5.

15. Hibbard JH, Stockard J, Mahoney ER, Tusler M. Development of the patient activation measure (PAM): conceptualizing and measuring activation in patients and consumers. Health Serv Res. 2004;39(4I):1005-1026.

16. Graffigna G, Barello S, Bonanomi A. The role of Patient Health Engagement model (PHE-model) in affecting patient activation and medication adherence: a structural equation model. PLoS One. 2017; 12(6):e0179865.

17. Gruman J, Rovner MH, French ME, et al. From patient education to patient engagement: implications for the field of patient education. Patient Educ Couns. 2010;78(3):350-356.

18. Forbat L, Cayless S, Knighting K, Cornwell J, Kearney N. Engaging patients in health care: an empirical study of the role of engagement on attitudes and action. Patient Educ Couns. 2009;74(1):84-90.

19. Hibbard JH, Mahoney E. Toward a theory of patient and consumer activation. Patient Educ Couns. 2010;78(3):377-381.

20. Rodriguez KM. Intrinsic and extrinsic factors affecting patient engagement in diabetes self-management: perspectives of a certified diabetes educator. Clin Ther. 2013;35:170-178.

21. Parker JL, Regan JF, Petroski J. Beneficiary activation in the Medicare population. Medicare Medicaid Res Rev. 2014;4(4):E14.

22. Ryvicker M, Feldman PH, Chiu Y-L, Gerber LM. The role of patient activation in improving blood pressure outcomes in Black patients receiving home care. Med Care Res Rev. 2013;70(6):636-652.

23. Rademakers J, Nijman J, Brabers AEM, de Jong JD, Hendriks M. The relative effect of health literacy and patient activation on provider choice in the Netherlands. Health Policy. 2014;114(2-3):200-206.

24. Nijman J, Hendriks M, Brabers A, de Jong J, Rademakers J. Patient activation and health literacy as predictors of health information use in a general sample of Dutch health care consumers. J Health Commun. 2014;19(8):955-969.

25. Lubetkin EI, Zabor EC, Brennessel D, Kemeny MM, Hay JL. Beyond demographics: differences in patient activation across new immigrant, diverse language subgroups. J Community Health. 2014;39(1):40-49.

26. Simmons LA, Wolever RQ, Bechard EM, Snyderman R. Patient engagement as a risk factor in personalized health care: a systematic review of the literature on chronic disease. Genome Med. 2014;6:16.

27. Gagliardi AR, Lemieux-Charles L, Brown AD, Sullivan T, Goel V. Barriers to patient involvement in health service planning and evaluation: an exploratory study. Patient Educ Couns. 2008;70(2):234-241.

28. Thompson AGH. The meaning of patient involvement and participation in health care consultations: a taxonomy. Soc Sci Med. 2007;64(6): 1297-1310.

29. Kennedy A, Gask L, Rogers A. Training professionals to engage with and promote self-management. Health Educ Res. 2005;20(5):567-578.

30. The King's Fund. Leadership and engagement for improvement in the NHS: together we can. Leadersh Rev. 2012:1-48.

31. Barello S, Guendalina G, Giuliana P, Maila M, Maurizio C, Livio P. An educational intervention to Train professional nurses in promoting patient engagement: a pilot feasibility study. Front Psychol. 2017:2020.

32. Coulter A. Patient engagement - what works? J Ambul Care Manage. 2012;35:80-89.

33. Barello S, Graffigna G, Vegni E, Savarese M, Lombardi F, Bosio AC. "Engage me in taking care of my heart": a grounded theory study on patient-cardiologist relationship in the hospital management of heart failure. BMJ Open. 2015;5(3):e005582.

34. Graffigna G, Barello S, Bonanomi A, Menichetti J. The motivating function of healthcare professional in eHealth and mHealth interventions for type 2 diabetes patients and the mediating role of patient engagement. J Diabetes Res. 2016;2016:2974521.

35. Zolnierek KBH, Dimatteo MR. Physician communication and patient adherence to treatment: a meta-analysis. Med Care. 2009;47:826-834.

36. Bigi S. Communication skills for patient engagement: argumentation competencies as means to prevent or limit reactance arousal, with an example from the italian healthcare system. Front Psychol. 2016;7: 1472 . 
37. Greene J, Hibbard JH, Alvarez C, Overton V. Supporting patient behavior change: approaches used by primary care clinicians whose patients have an increase in activation levels. Ann Fam Med. 2016;14(2): $148-154$.

38. Barello S, Graffigna G. Engaging patients to recover life projectuality: an Italian cross-disease framework. Qual Life Res. 2015;24(5):1087-1096.

39. Lamiani G, Barello S, Browning DM, Vegni E, Meyer EC. Uncovering and validating clinicians' experiential knowledge when facing difficult conversations: a cross-cultural perspective. Patient Educ Couns. 2012; 87(3):307-312.

40. Couët N, Desroches S, Robitaille H, et al. Assessments of the extent to which health-care providers involve patients in decision making: a systematic review of studies using the OPTION instrument. Health Expect. 2013;14:542-561.

41. Koh HK, Brach C, Harris LM, Parchman ML. A proposed "health literate care model" would constitute a systems approach to improving patients' engagement in care. Health Aff (Millwood). 2013;32(2):357-367.

42. Grant B, Colello SH. Culture change through patient engagement. Nurs Manage. 2010;41(10):44-46.

43. Appelboom G, LoPresti M, Reginster J-Y, Sander Connolly E, Dumont EPL. The quantified patient: a patient participatory culture. Curr Med Res Opin. 2014:1-3.

44. Zeuner R, Frosch DL, Kuzemchak MD, Politi MC. Physicians' perceptions of shared decision-making behaviours: a qualitative study demonstrating the continued chasm between aspirations and clinical practice. Health Expect. 2014;18(6):2465-2476.

45. Abdelhadi N, Drach-Zahavy A. Promoting patient care: work engagement as a mediator between ward service climate and patient-centred care. J Adv Nurs. 2012;68:1276-1287.

46. Gill PS. Patient engagement: an investigation at a primary care clinic. Int J Gen Med. 2013;6:85-98.

47. Piña IL, Cohen PD, Larson DB, et al. A framework for describing health care delivery organizations and systems. Am J Public Health. 2015; 105(4):670-679

48. Safran DG, Miller W, Beckman H. Organizational dimensions of relationship-centered care. Theory, evidence, and practice. J Gen Intern Med. 2006;21(Suppl 1):S9-S15.

49. Luxford K, Safran DG, Delbanco T. Promoting patient-centered care: a qualitative study of facilitators and barriers in healthcare organizations with a reputation for improving the patient experience. Int J Qual Health Care. 2011;23(5):510-515.

50. Hibbard JH. Community-based participation approaches and individual health activation. J Ambul Care Manage. 2009;32(4):275-277.

51. Bertoni A, Donato S, Graffigna G, Barello S, Parise M. Engaged patients, engaged partnerships: singles and partners dealing with an acute cardiac event. Psychology, health \& medicine. 2015;20(5):505-517.

52. Grande SW, Faber MJ, Durand MA, Thompson R, Elwyn G. A classification model of patient engagement methods and assessment of their feasibility in real-world settings. Patient Educ Couns. 2014;95(2):281-287.

53. Ng C-J, Lee P-Y, Lee Y-K, et al. An overview of patient involvement in healthcare decision-making: a situational analysis of the Malaysian context. BMC Health Serv Res. 2013;13:408.

54. Fisher J, Clayton M. Who gives a tweet: assessing patients' interest in the use of social media for health care. Worldviews Evid Based Nurs. 2012; 9(2):100-108.

55. Graffigna G, Barello S, Triberti S, Wiederhold BK, Bosio AC, Riva G. Enabling ehealth as a pathway for patient engagement: a toolkit for medical practice. Stud Health Technol Inform. 2014;199:13-21.

56. Irizarry T, DeVito Dabbs A, Curran CR. Patient portals and patient engagement: a state of the science review. J Med Internet Res. 2015; 17(6):e148.

57. Franklin VL, Greene A, Waller A, Greene SA, Pagliari C. Patients' engagement with "Sweet Talk" - a text messaging support system for young people with diabetes. J Med Internet Res. 2008;10(2):e20.
58. Hibbard J, Helen G. Supporting people to manage their health: an introduction to patient activation. King's Fund. 2014;51:1-51.

59. Duke CC, Lynch WD, Smith B, Winstanley J. Validity of a new patient engagement measure: the altarum consumer engagement (ACE) measure $^{\mathrm{TM}}$. Patient. 2015;8(6):559-568.

60. Graffigna G, Barello S, Triberti S. Giving (back) a role to patients in the delivery of healthcare services: Theoretical roots of patient engagement. Patient Engagement: A Consumer-Centered Model to Innovate Healthcare. Graffigna G, Barello S, Triberti S editors. Warsaw: De Gruyter Open; 2015:13-26.

61. Coulter A, Collins A. Making decision making a reality. No decision about me, without me. Kings Fund. 2011:1-56. Available from: http://www.kingsfund.org.uk/publications/nhs_decisionmaking.html. Accessed March 23, 2018.

62. Coulter A. Measuring what matters to patients. BMJ. 2017:j816.

63. Alba JW, Hutchinson JW. Consumer psychology. In: Handbook of Applied Cognition, Second Edition. New York: Wiley; 2008:683-711.

64. Twedt DW. Consumer psychology. Annu Rev Psychol. 1965;16(1): 265-294.

65. Graffigna G, Barello S, Libreri C, Bosio CA. How to engage type-2 diabetic patients in their own health management: implications for clinical practice. BMC Public Heal. 2014;14(1):648.

66. Graffigna G, Barello S, Bonanomi A, Riva G. Factors affecting patients' online health information-seeking behaviours: the role of the Patient Health Engagement (PHE) Model. Patient Educ Couns. 2017; 100(10):1918-1927.

67. Graffigna G, Barello S. Modelling patient engagement in healthcare: Insight for research and pratice. Patient Engagement: A ConsumerCentered Model to Innovate Healthcare. Graffigna G, Barello S, Triberti S editors. Warsaw: De Gruyter Open; 2015:27-43.

68. McCormack L, Thomas V, Lewis MA, Rudd R. Improving low health literacy and patient engagement: a social ecological approach. Patient Educ Couns. 2017;100(1):8-13.

69. Kubler-Ross E. On Death and Dying. Vol 1. New York: The Macmillan Company; 1969.

70. Prochaska JO, Velicer WF. The transtheoretical model of health behavior change. Am J Heal Promot AJHP. 1997;12(1):38-48.

71. Graffigna G, Barello S, Bonanomi A, Lozza E. Measuring patient engagement: development and psychometric properties of the patient health engagement (PHE) scale. Front Psychol. 2015;6:274.

72. Graffigna G, Barello S. The Value of Measuring Patient Engagement in Healthcare: New Frontiers for Healthcare Quality. Graffigna G, editor. Promoting Patient Engagement and Participation for Effective Healthcare Reform, Medical Information Science Reference. Hershey PA, USA: 2016:192-214.

73. Zhang Y, Graffigna G, Bonanomi A, et al. Adaptation and validation of a Chinese version of patient health engagement scale for patients with chronic disease. Front Psychol. 2017;8:1-9.

74. Magallares A, Graffigna G, Barello S, Bonanomi A, Lozza E. Spanish adaptation of the Patient Health Engagement scale (S.PHE-s) in patients with chronic diseases. Psicothema. 2017;29(3):408-413.

75. Menichetti J, Graffigna G. "PHE in action": development and modeling of an intervention to improve patient engagement among older adults. Front Psychol. 2016;7:1405.

76. Menichetti J, Graffigna G. "PHE in Action": development and modeling of an intervention to improve patient engagement among older adults. Front Psychol. 2016;7:1405.

77. Légaré F, Witteman HO. Shared decision making: examining key elements and barriers to adoption into routine clinical practice. Health affairs. 2013;32(2):276-284.

78. Mittler JN, Martsolf GR, Telenko SJ, Scanlon DP. Making sense of "consumer engagement" initiatives to improve health and health care: a conceptual framework to guide policy and practice. The Milbank Quarterly. 2013;91(1):37-77. 
Patient Preference and Adherence

Dovepress

\section{Publish your work in this journal}

Patient Preference and Adherence is an international, peer-reviewed, open access journal that focuses on the growing importance of patient preference and adherence throughout the therapeutic continuum. Patient satisfaction, acceptability, quality of life, compliance, persistence and their role in developing new therapeutic modalities and compounds to optimize

Submit your manuscript here: http://www.dovepress.com/patient-preference-and-adherence-journ clinical outcomes for existing disease states are major areas of interest for the journal. This journal has been accepted for indexing on PubMed Central The manuscript management system is completely online and includes a very quick and fair peer-review system, which is all easy to use. Visit http://www. dovepress.com/testimonials.php to read real quotes from published authors. 